\title{
Das Ich im Fokus. Sprecher-Inszenierungen in den geistlichen Liedern Oswalds von Wolkenstein
}

\section{Einleitung: Wer spricht?}

,Ich` zu sagen ist nicht die einzig denkbare Sprechhaltung im geistlichen Lied. ${ }^{1}$ Blickt man auf die lateinische liturgische Lieddichtung, wird man womöglich sogar postulieren können, dass hier die Inszenierung der Sprecher-Instanz als Kollektiv überwiegt. In der Regel artikuliert sich also ein Wir, das von der Schola im gesanglichen Vortrag gefüllt wird, aber über die Sänger hinaus die anwesende Gemeinde und die gesamte Gemeinschaft der Gläubigen umfasst. Die Inszenierung der Sprecher-Instanz als kollektives Wir findet sich auch im geistlichen Euvre Oswalds von Wolkenstein. Oswald wählt die ,wir'-Form insbesondere in solchen Fällen, in denen eine enge Anbindung an die liturgische Liedtradition oder an rituelle Gebetsformen besteht. ${ }^{2}$ Ganz in der , wir'-Form gehalten sind etwa die beiden Übertragungen des Mundi renovatio (Kl. 129) und des Mittit ad virginem (Kl. 130) sowie das Benedicte (Kl. 14) und die beiden Kalendergedichte (Kl. 28, Kl. 67). ${ }^{3}$ Die Entscheidung folgt offenbar funktionalen Gesichtspunkten: Die , wir'Form fungiert als Gattungssignal.

Weitaus typischer für Oswalds geistliches Liedschaffen ist freilich, dass das Wir zumindest phasenweise durch ein Ich abgelöst wird; die allermeisten seiner geistlichen Lieder sind sogar durchgängig in der ersten Person Singular ge-

1 Ähnlich wie Haferland unterscheide ich zwischen dem Ich als Begriff für die Sprecher-Instanz und ,ich', wenn das Personalpronomen gemeint ist. Vgl. Haferland, S. 197, Anm. 71.

2 Die eindeutige Zuordnung zu der Gruppe der weltlichen oder zu der der geistlichen Lieder fällt bei Oswald nicht immer ganz leicht. Welche Lieder als geistlich angesehen werden, kann daher variieren. Vgl. die entsprechenden Gruppenzuweisungen bei Spechtler: Beiträge II, S. 272-279; Wachinger: Art. ,Oswald‘, Sp. 159-162; Spicker, S. 86-118; Löser: Geistliche Lieder; Löser: Geistliche Dichtung. Ich zähle folgende Lieder zu den geistlichen Liedern: Kl. 1-15, Kl. 22-24, Kl. 27-29, Kl. 31, Kl. 32, Kl. 34-36, Kl. 38-40, Kl. 67, Kl. 78, Kl. 95, Kl. 109a/b, Kl. 111-115, Kl. 118, Kl.126, Kl. 129, Kl. 130 und Kl. 134. Referenzpunkt ist die Gesamtausgabe der Lieder von Klein (Die Lieder Oswalds), deren Zählung übernommen wird. Die Lieder werden jedoch nach der Auswahlausgabe von Wachinger (Oswald: Lieder) zitiert, wenn sie in ihr enthalten sind. In diesem Fall wird durch das Kürzel Wa. auf die Ausgabe verwiesen.

3 Durchgängig in der ,wir'-Form abgefasst sind neben den genannten Liedern Kl. 14, Kl. 28, Kl. 67, Kl. 129 und KL. 130 noch die Lieder Kl. 38, Kl. 109a/b, Kl. 113 und Kl. 134.

Ә OpenAccess. (C) 2020 Britta Bußmann, publiziert von De Gruyter. (cc)BY Dieses Werk ist lizenziert unter der Creative Commons Attribution 4.0 International. https://doi.org/10.1515/9783110666816-009 
schrieben. Formal artikuliert sich in diesen Liedern eine Einzelstimme, die zumindest potenziell auf einen konkreten Sprecher verweist. Anders als für das Wir stellt sich für das Ich mithin die Frage, wer hier eigentlich spricht. In der OswaldForschung ist sie vorwiegend als Frage nach der biographischen Referentialisierbarkeit des Ich behandelt worden. Die Diskussion stützt sich deswegen vornehmlich auf die explizit mit biographischen Elementen arbeitenden Lieder. Zur Debatte steht dann nicht die grundsätzliche Verweisrichtung des Ich, das sich in diesem Kontext immer auf die Urheber-Instanz bezieht und in einem Teil der Lieder sogar direkt als ,Wolkenstein' oder ,Wolkensteiner' benannt ist. ${ }^{4}$ Fraglich ist vielmehr, ob das Ich biographisch als historischer Oswald oder, wie es sich in der jüngeren Forschung durchzusetzen scheint, als literarisch vermittelte Autorkonkretisation aufzulösen ist, d.h. als Oswald-Persona. ${ }^{5}$

Der mögliche Bedeutungsrahmen des Ich ist damit allerdings noch nicht in seiner Gesamtheit ausgeschöpft. Ist das Ich zwar als Stimme präsent, doch fehlt ihm eine biographische Konkretisierung, so ist es nicht zwingend notwendig, das Ich aus dem Werkkontext heraus als Oswald-Persona $\mathrm{zu}$ identifizieren. ${ }^{6}$

4 Namenssignaturen haben zwölf der insgesamt 43 geistlichen Lieder (Kl. 1, Kl. 3, Kl. 4, Kl. 6, Kl. 7, Kl. 27, Kl. 35, Kl. 39, Kl. 111, Kl.112, Kl. 115, Kl.118), wobei die Selbstnennungen in Kl. 3 und in Kl. 115 in der dritten Person Singular formuliert sind. In weiteren sieben Liedern verwendet Oswald biographische Details, um die Sprecher-Instanz auszugestalten, ohne dass das Ich seinen Namen tragen würde (Kl. 2, Kl. 5, Kl. 9, Kl. 12, Kl. 23, Kl. 24, Kl. 36).

5 Vgl. etwa Philipowski, S. 340. Zum Begriff der Autorkonkretisation vgl. Hausmann, S. 26-36. Er bezeichnet die von den Texten evozierte Vorstellung, die sich die Rezipientinnen und Rezipienten über das Subjekt der Textaussage, d.h. über die Urheber-Instanz, machen (vgl. ebd., S. 26). Der Begriff der Autorkonkretisation, so wie Hausmann ihn benutzt, setzt dabei einen zweidimensionalen Fiktionalitätsbegriff voraus: „Neben die Relation textinternes Ich - rezipientenseitige Autorkonkretisation tritt die Frage nach der externen Referentialisierbarkeit, also etwa die Frage nach dem Verhältnis von Autorkonkretisation und tatsächlichem Autor bzw. anwesendem Sprecher“ (ebd., S. 98). Bezogen auf Oswalds Lieddichtung heißt das: Ähnlich wie in hochminnesängerischen Mannesliedern fällt in Oswalds (pseudo-)autobiographischen Liedern schon allein aufgrund der dargestellten Sprechsituation (Reflexionslieder, in denen das Ich über sich spricht) das Ich mit der Autorkonkretisation zusammen. Die biographischen Elemente - und hier liegt das entscheidend Neue - sorgen dann zusätzlich dafür, dass sich der Unterschied zwischen Autorkonkretisation und textexternem, realen Autor minimiert, ohne allerdings je ganz zu schwinden. Wie Toepfer am Beispiel von Kl. 3, Kl. 33 und Kl. 39 zeigt, setzt Oswald in einigen Liedern Selbstironie als Mittel ein, um die Gleichsetzung von Sprecher-Ich und historischem Autor als vorschnelle Deutung zu entlarven. Vgl. Toepfer, insbes. S. 229f. und S. $236 \mathrm{f}$.

6 In der Forschung wird das z.T. unumwunden gemacht. So setzt Spechtler für das Ich von solchen Marienliedern, die Elemente des Minnesang nutzen, ohne jede Diskussion voraus, dass hier die Oswald-Persona spricht: „Was Oswald nun in Steigerung dieses Phänomens so weit ausnützt, einerseits den Ich-Bezug (Ich-Oswald, also das autobiographische Element seiner weltlichen Lyrik) auch in die Marienlieder einzuführen“ (Spechtler: Beiträge III, S. 194f.). 
Schließlich zeigt bereits die Existenz der in der , wir'-Form gehaltenen Lieder, dass Oswald die Sprecher-Instanz der geistlichen Lieder durchaus variiert. Gerade wenn man die Konventionen der Gattung des geistlichen Lieds zur Basis der Überlegungen macht, liegt in Fällen, in denen das Ich vage konturiert, gleichsam unkörperlich bleibt, die Vermutung näher, dass das Ich durch die Rezipienten gefüllt werden soll. Es ist, folgert Harald Haferland mit Blick auf die Gattung, nämlich „offensichtlich, daß ein Dichter von Gebeten oder Andachtsliedern dem Nutzer eine sprachliche Form zuspielt, die ihm auch das ,ich“ überläßt“. Der Nutzer kann „das ,ich` auf sich beziehen, und der Dichter hat es schon darauf zugeschnitten“. ${ }^{7}$ Die ,ich'-Form dient im geistlichen Kontext folglich nicht immer schon der Festlegung auf einen konkreten Sprecher, sondern der Regulation der Sprechsituation, indem sie suggeriert, dass der jeweilige Rezipient oder die jeweilige Rezipientin sich in einer intimen Eins-zu-eins-Kommunikation an die im Lied angerufene Instanz (Gott, Maria, Heilige) wendet.

Dass eine solch offene, letztlich auf die Übernahme durch die Rezipientinnen und Rezipienten abzielende Ich-Konzeption auch für Oswalds geistliche Lieder eine Rolle spielen könnte, hat bislang vor allem Franz Viktor Spechtler angemerkt. Im Zusammenhang mit der von ihm vorgenommenen Gruppierung der geistlichen Lieder in ,subjektive“ geistliche Lieder, bei denen „Elemente und Traditionen der geistlichen Lyrik [...] eine Funktion innerhalb des ,Autobiographischen“" erfüllen, ${ }^{8}$ und ,objektive“ geistliche Lieder, für die das nicht gilt und die stattdessen „,in geistlichen Liedtraditionen stehen“, ${ }^{9}$ weist er auf die unterschiedliche Ausformung der Sprecher-Instanzen in den beiden Liedgruppen hin: „Hinter dem Ich der ,subjektiven“ geistlichen Lieder ist ein Individuum, der Sänger etwa, vorstellbar, hinter dem der ,objektiven“ das kollektive Ich (bzw. das Wir) der Sünder, Christen etc. "10 Anknüpfend an diesen Befund, den Spechtler selbst nicht weiter verfolgt hat, rückt der vorliegende Beitrag die Frage nach der relativen Offenheit bzw. Geschlossenheit der Ich-Position gegenüber den Rezipientinnen und Rezipienten ins Zentrum der Auseinandersetzung mit Oswalds geistlichen Liedern. Ziel des Unternehmens ist es, den Blick auf die in Oswalds Liedern vorzufindenden Gestaltungsmöglichkeiten und Funktionen des Ich-Sprechers zu weiten und dies nicht zuletzt dadurch, dass dem (pseudo-)autobiographischen Ich aus dieser Perspektive nicht von vornherein schon ein Sonderstatus zugewiesen wird: Biographische Konkretisierung ist zunächst einmal nur eins der

7 Haferland, S. 197.

8 Spechtler: Beiträge II, S. 277.

9 Spechtler: Beiträge III, S. 182. Vgl. überdies ders.: Beiträge II, S. 277.

10 Ebd., S. 276, Anm. 8. 
denkbaren Mittel, das Ich als geschlossen zu konzipieren und eine Besetzung der Ich-Position durch die Rezipientinnen und Rezipienten zu verhindern.

\section{Das Ich in Oswalds geistlichen Liedern}

Hinsichtlich ihrer Ausbildung der Ich-Instanz bilden Oswalds geistliche Lieder keinen monolithischen Block. Die Forschung hat dies schon lange gesehen, münzt solche Aussagen jedoch vorwiegend auf die verschiedenen Sprecherrollen, die das Ich einnimmt, indem es sich beispielsweise als Ratgeber, als Sünder und dem Tode Naher oder als Maria innig verbundener Gläubiger geriert. Die Varianz betrifft indes auch die Deutlichkeit, mit der das Ich konturiert wird, und die Ausschließlichkeit, mit der Ich und Autorkonkretisation gleichzusetzen sind. Vor allem ist keineswegs davon auszugehen, dass - wie Spechtlers oben zitierter Befund es anzudeuten scheint - lediglich eine einfache Schwarz-Weiß-Unterscheidung zwischen einem (pseudo-)autobiographisch gemeinten Ich auf der einen und einem ,kollektiven“ Ich auf der anderen Seite existiert und dass diese Verteilung einhergeht mit der inhaltlich begründeten Trennung in ,subjektive، und ,objektive' geistliche Lieder. Bestes Beispiel hierfür ist das Beichtlied Mein sünd und schuld eu, priester, klag (Kl. 39/Wa. 35). Spechtler rechnet es, ohne dies weiter zu kommentieren, zu den ,objektiven' geistlichen Liedern. ${ }^{11}$ Ausschlaggebend für diese Zuordnung ist wahrscheinlich der Umstand, dass Oswald Kl. 39/ Wa. 35 zumindest in den Anfangsteilen als eine „Musterbeichte“ anlegt, inhaltlich also stereotype Formeln aus standardisierten Beichtkatalogen oder Beichtspiegeln repetiert. ${ }^{12}$ Doch das Ich, das hier seine Verfehlungen bekennt (etwa: An dem gelouben zweifel ich; I,9), ist nur auf den ersten Blick ein ,kollektives‘: Sprechsituation und Sprecher-Instanz des Liedes sind vielmehr ausgehend von der Namensnennung (Wolkenstainer; V,7) und dem poetologischen Kommentar der fünften Strophe zu bestimmen (aus beichten solt ich leren / Durch mein gesangk vil hoveleut; V,8f.). Vor diesem Hintergrund erweist sich das Sünder-Ich des Liedanfangs als bloße Pose des ,Wolkensteiner'-Ich, das durch die Namensnennung personal zugespitzt und auf die Autorkonkretisation zu beziehen ist. ${ }^{13}$ Notwendig

11 Vgl. ebd., S. 278. Zum Lied vgl. Spicker, S. 95; Löser: Geistliche Dichtung, S. 21; Toepfer, S. $234-$ 236; Kraß.

12 Löser: Geistliche Dichtung, S. 21.

13 Oswald legt in dem Lied also weder eine ernst gemeinte Lebensbeichte ab noch ermöglicht er den Rezipientinnen und Rezipienten dies zu tun, denn dafür müsste das Lied ein stabiles ,kollektives' Ich besitzen. Er reflektiert stattdessen die Funktion der Gattung des Beichtlieds. Dieses 
ist demzufolge eine feinere Unterteilung, die sich zudem allein auf die Gestaltung der Ich-Instanz im jeweiligen Lied stützt.

Überblickt man Oswalds geistliche Lieder, dann lassen sich im Hinblick auf die relative Geschlossenheit bzw. Offenheit der Ich-Darstellung mindestens vier unterschiedliche Typen unterscheiden, die ich im Weiteren vorstellen möchte. Ich werde die vier Typen aufsteigend hinsichtlich des Grads der Geschlossenheit der Ich-Position besprechen. Maßstab für diese Einteilung sind dabei folgende Merkmale, welche in meinen Augen Mindestbedingungen formulieren, um ein Ich als offen gestaltet definieren zu können: die geringe Konturierung der Sprecherrolle, die Allgemeingültigkeit der vom Ich vertretenen Meinungen und das Fehlen einer weiteren, angeredeten Instanz, die als Auffangpunkt für die Rezipientinnen und Rezipienten gestaltet sein könnte. ${ }^{14}$ Liegen diese Faktoren vor, kann man die Sprecher-Instanz als offen bezeichnen; sie kann dann also von den Rezipientinnen und Rezipienten ausgefüllt werden.

Zur Illustration des je gemeinten Typs wähle ich jeweils ein Lied Oswalds aus. So tritt das Gracias (Kl. 15/Wa. 36b) für den ersten Typ ein, das Marienlied Es leucht durch grau die fein lasur (Kl. 34/Wa. 39) für den zweiten, das zu diesem Lied melodiegleiche, auf die Weihnachtszeit bezogene Lied In Suria ain braiten hall (Kl. 35/Wa. 37) für den dritten und schließlich das Lied Ain anefangk (Kl. 1/Wa. 22) für den vierten. ${ }^{15}$

\subsection{Das Ich als Exponent des Wir}

Unter dem ersten Typ fasse ich solche Lieder, in denen das Ich nur partienweise hinter dem Wir hervortritt. Die erste Person Plural ist in diesen Liedern mithin die vorherrschende Äußerungsform der Sprecher-Instanz, so dass diese grundsätzlich als Kollektiv gestaltet ist. ${ }^{16}$

Verständnis von Kl. 39/Wa. 35 stützt sich im Wesentlichen auf die Interpretation von Kraß und Toepfer; vgl. Kraß, insbes. S. 65 f. und Toepfer, S. 234-236.

14 Ich orientiere mich für diese Faktoren an Philipowskis Überlegungen zum ebenfalls offen gestalteten Ich der Minnereden: Dieses zeichnet sich auch durch die Allgemeingültigkeit der geäußerten Positionen und durch fehlende Konkretisierung (hier: Namenlosigkeit) aus. Vgl. Philipowski, S. 326-330.

15 Das Verfahren lehnt sich an Brauns Versuch an, den biographischen Gehalt von Personennamen im Werk von Oswald zu klassifizieren. Leitfrage ist dabei, wie notwendig biographisches Wissen ist, um die Anspielungen entschlüsseln zu können. Vgl. Braun, insbes. S. 140 - 143.

16 Hierzu gehören die Lieder Kl. 13, Kl. 15, Kl. 29 und Kl. 126. Das Lied Kl. 29 ist in der Fassung der Hs. A (Wien, ÖNB, cod. 2777, fol. $37^{\circ}$ ) in die ,ich'-Form umgeschrieben (vgl. Spechtler: Beiträge II, S. 278). In dieser Fassung wäre es dem zweiten Typ zuzuweisen. 
Ein Beispiel für diesen Typ ist das dreistrophige Danklied Wol auf, als das zue himel sei (Kl. 15/Wa. 36b). ${ }^{17}$ Es beginnt mit einer vom kollektiven Wir an die Engel und Heiligen (als das zue himel sei; I,1) herangetragenen Bitte, den Gläubigen bei ihrer an Gott gerichteten Danksagung zu helfen: und helft uns sagen im den dank $(I, 4)$. Erst in der zweiten Strophe löst sich erstmals ein Ich aus der Gemeinschaft der Gläubigen heraus. Dieses wendet sich direkt an die Gottesmutter (frau; II,1) und beklagt seine Sündhaftigkeit:

$$
\begin{aligned}
& \text { vor dem ich sünder mich beklag, } \\
& \text { das ich in elendlicher wag } \\
& \text { vil han verzert unnützer tag } \\
& \text { in diser snöden zeit so brait, } \\
& \text { die mir verlech dein kind. (II,4-8) }
\end{aligned}
$$

Die abschließende dritte Strophe mit ihrer Bitte um Hilfe in der Todesstunde weist dann sowohl ,ich'- als auch ,wir'-Nennungen auf, wobei diese sich abwechseln:

ich ruef in angestlicher wat:

hilf, magt, mit ganzer trinitat

und las uns nicht der helle vas!

so pistus, frau, der ich genas.

des sing wir deo gracias. (III,2-6)

Inhaltlich lässt sich beobachten, dass die Engführung der Sprecher-Instanz, d. h. der Wechsel vom Wir zum Ich, mit einer thematischen Erweiterung einhergeht. Ab Strophe II lässt Oswald den engen Rahmen des Tischgebets hinter sich. Lob und Dank gelten von da an allgemein dem Heilshandeln und der Hilfe, die das Ich und die Gläubigen durch Gott, Christus, vor allem aber durch Maria bereits erfahren haben und auf die sie auch in Zukunft hoffen. Erst diese inhaltliche Erweiterung macht es überhaupt möglich, die vom Ich vorgetragenen Gedanken (Sündenangst, Bitte um Erlösung) im Kontext des Gracias zu äußern.

Grundsätzlich ist die Ich-Position innerhalb des Lieds offen gezeichnet, denn die in der ersten Person Singular abgefassten Aussagen bleiben zumindest ihrem generellen Inhalt nach konventionell. Das Bekenntnis der eigenen Sündhaftigkeit und die Hinwendung an die gnädige Gottesmutter, von der das Ich sich Hilfe erhofft, sind schließlich beides typische Elemente der spätmittelalterlichen Marienfrömmigkeit. Dies verhindert eine Identifikation des Ich mit der Autorkonkretisation nicht schon von vornherein, legt sie aber auch nicht in besonderer Weise

17 Zum Lied vgl. Spechtler: Beiträge II, S. 279-284; Spicker, S. 92; Löser: Geistliche Dichtung, S. $11 \mathrm{f}$. 
nahe - im Gegenteil: Wollte man diese Deutungsoption verabsolutieren, wollte man also die Sündenklage der Strophe II und die Hilfebitte der Strophe III ausschließlich als Hervortreten der Oswald-Persona lesen, benötigte man textuelle Faktoren, die eine solche Interpretation stützen. Ein biographischer Hintergrund der ,ich'-Aussagen scheint allerdings nicht zwingend gegeben. So ist es zwar unter Umständen möglich, die Erwähnung der elendliche[n] wag (II,5) als Referentialisierung von Oswalds Reisen zu vereindeutigen. Das Attribut elendliche müsste dann mit ,fremd' übersetzt werden und sich direkt auf die von Oswald durchquerten fremden Länder beziehen. ${ }^{18}$ Überzeugender scheint mir freilich Marolds Vorstoß, elendliche im Anschluss an Kl. 9,I,12 (nu muess ich wachen, seufzen, zittren ellentleich) mit, jämmerlich‘ und wag mit ,Bewegung` zu übertragen: Die Fügung bezöge sich in diesem Fall allgemein auf das Erdenleben, das als jämmerliches Hin-und-Her-Treiben verbildlicht würde. ${ }^{19}$

Einer solch allgemeineren Lesart des Verses entspricht, dass sich das Ich im direkten Zusammentreffen mit dem Wir gerade nicht als eigenständige, gegenüber dem Wir ausdifferenzierte Sprechposition erweist. Die persönliche Bitte des Ich um Hilfe und Vergebung und sein persönlicher Dank gehen letztlich jeweils in der gemeinsamen Bitte und im gemeinsamen Dank auf. Wie gleitend dabei die Übergänge gestaltet sind, zeigt sich bereits in der ersten in Strophe III enthaltenen Bitte (III,3f.). Weil die Redeeinleitung das Ich als Sprecher (ich ruef; III,2) aufbietet, könnte sich der Beginn der Bitte (hilf, magt, mit ganzer trinitat; III,3) auf dieses zurückbeziehen. Die Fortführung (und las uns nicht der helle vas; III,4) verdeutlicht indes, dass das Ich hier stellvertretend für die gesamte Gemeinschaft der Gläubigen spricht. Diese enge Verbindung zwischen Ich und Wir setzt sich fort. Im nächsten Vers III,5 wird Maria zur Retterin speziell des Ich erklärt (so pistus, frau, der ich genas), doch den Dank dafür artikuliert das Wir (des sing wir deo gracias; III,6).

Letztlich kann man schließen: Das Ich der Lieder vom ersten Typ ist, da es nur punktuell zu Wort kommt, eher vage konturiert, es entwickelt kein eigenständiges Profil gegenüber dem Wir und agiert gleichsam als dessen Verlängerung oder Exponent. Typisch ist das Hinübergleiten zwischen beiden Sprechpositionen, so dass auch aus diesem Grund das Ich nicht als ein gesonderter Standpunkt erfahrbar wird: Das Ich bleibt stets im Kontakt mit der Gruppe. Das Ich öffnet sich demzufolge gegenüber den Rezipientinnen und Rezipienten. In Kl. 15/Wa. 36b kann dadurch ein jeder Rezipient das zentrale Heilsversprechen des Textes -

18 Offenbar ist das bei Wachinger angedacht, denn dieser übersetzt elendliche[ ] wag mit ,Waagschale der Fremde‘; vgl. Oswald: Lieder, S. 279.

19 Vgl. Marold, S. 167. Hofmeister schlägt als Übersetzung ,erbärmliches Treiben` vor; vgl. Oswald: Sämtliche Lieder, S. 47. 
nämlich die Erfüllung der Bitte um Erlösung - nicht nur als Teil einer Glaubensgemeinschaft, sondern individuell formulieren und auf diese Weise direkt auf sich beziehen. ${ }^{20}$

\subsection{Das Ich als Hohlform}

Zum zweiten Typ rechne ich solche Lieder, in denen das Ich zwar ausschließlich oder zumindest hauptsächlich - spricht, dabei aber wie bei den Ich-Nennungen des ersten Typs eher vage konturiert ist. Ihm fehlen spezifische Züge, die eine Zuordnung zu einem bestimmten Sprecher notwendig machen würden. Dennoch ist das Ich dieser Lieder als Einzelstimme deutlich präsent; die Hinwendung an die angerufene Instanz ist durch die (fast) durchgängig gewahrte ,ich'-Form als persönliche Anrede an Gott oder an die Gottesmutter inszeniert. Maria erscheint in fast allen Liedern dieses Typs als Angesprochene. ${ }^{21}$ Diese Art von Ich kann man vielleicht am ehesten mit dem von Katharina Philipowski im Anschluss an Ingeborg Glier eigentlich mit Blick auf die Sprecher-Instanz von Minnereden genutzten Begriff der ,Hohlform‘ beschreiben. Philipowski versteht darunter „eine Position innerhalb des Textes, die dazu konzipiert ist, vom jeweiligen Rezipienten eingenommen zu werden“.22 Auch die Minnereden-Ichs können eigenes Erleben vortragen, ohne selbst eine eigenständige Figur zu sein. Sie dienen als Platzhalter für die Rezipientinnen und Rezipienten und sichern deren Partizipationsmöglichkeit. ${ }^{23}$

Mit dem an Maria gerichteten Wecklied Es leucht durch grau die fein lasur (Kl. 34/Wa. 39) habe ich mich für ein Beispiellied entschieden, dessen Originalität

20 Bei den Liedern dieses Typs ist es denkbar, dass die Entscheidung über die Referentialisierung des Ich Auswirkungen darauf hat, wie man die Sprechsituation der Lieder beurteilt. Liest man das Ich als ,kollektives‘ Ich, geht man wahrscheinlich davon aus, dass das Lied von einer Gruppe gesprochen wird, aus der sich in Einzelfällen ein Ich herauslöst. Setzt man das Ich doch (was in meinen Augen allerdings aufgrund der geringen Eigenständigkeit der Ich-Position die nachgeordnete Lesart ist) mit der Autorkonkretisation gleich, würde man die Sprechsituation wahrscheinlich so auffassen, dass das Ich immer spricht, sich aber partienweise zum Sprachrohr einer Gemeinschaft macht und dann ,wir' sagt.

21 In diese Gruppe gehören die Lieder Kl. 31, Kl. 34, Kl.40 und Kl.78. Zählt man Kl. 37 und Kl. 120 (so bei Löser: Geistliche Dichtung, S. 16f. im Anschluss an Hartmann: Madonnenbilder, S. 72) als Marienlieder und nicht als Frauenpreis, gehören sie ebenfalls hierher. Bis auf Kl. 31 sind also alle Lieder dieser Gruppe Marienlieder.

22 Philipowski, S. 329. Vgl. überdies Glier, S. 394-399.

23 Vgl. Philipowski, S. 329. 
in der Forschung zu Recht betont wird. ${ }^{24}$ Burghart Wachinger hebt die Kühnheit hervor, mit der Oswald die sonst üblichen Grenzen der Erotisierung der Marienfrömmigkeit übersteigt, indem er Maria zur Geliebten des Ich stilisiert. ${ }^{25}$ Gestaltet wird demzufolge eine sehr intime Situation: Das offenbar zuerst erwachte Ich beschreibt den Tagesanbruch (Es leucht durch grau die fein lasur; I,1) und wendet sich an die neben ihm liegende Dame mit der Aufforderung, ebenfalls nach draußen zu blicken (plick durch die prau; I,3). ${ }^{26}$ Dieses Arrangement setzt voraus, dass das Ich und die Angeredete, welche erst in Strophe II mit letzter Sicherheit als Maria identifiziert wird (die uns gepar / ein frucht keuschlich zu freuden; II,9f.), die Nacht miteinander verbracht haben. ${ }^{27}$ Dass Maria als Minnedame erscheint, prägt auch die Bittformeln des Ich. Dieses wünscht sich in Strophe I, von der Geliebten gegrüßt zu werden (wurd mir zu teil / von ir ain freuntlich grüesslin; I,7 f.); ${ }^{28}$ in der Schlussbitte spricht das Ich Maria direkt als ,Liebste“ an:

\footnotetext{
Ste für die tür grausleicher not, wenn sich mein haupt wirt senken gen deinem feinen mündlin rot, so tue mich, lieb, bedenken! (III,9-12)
}

Individualität der Themenwahl meint aber nicht zugleich schon Individualität des hier fast durchgängig ${ }^{29}$ agierenden Ich. Über die Sprecher-Instanz erfährt man wenig mehr, als dass es sich - folgt man heteronormativen Konventionen - um einen Mann handelt, da Maria und das Ich ein Liebespaar sind. Inhaltlich verbleiben die Selbstaussagen des Ich im Formelhaften. Das in der ersten Strophe geäußerte Bekenntnis, dass niemand Maria vollständig loben könne (dem niemant kan nach meim verstan / plasiniren neur ain füesslin; I,5f.), gehört zum selbstverständlichen Repertoire der Gattung Marienlob und ist trotz des auffälligen Lehnworts plasiniren ${ }^{30}$ so allgemein formuliert, dass ein poetologisches

24 Vgl. zum Lied Spechtler: Beiträge III, S. 193f.; Hartmann: Einheit; Spicker, S. 87; Wachinger: Blick, S. 302-310; Löser: Geistliche Dichtung, S. $18 \mathrm{f}$.

25 Wachinger: Blick, S. 304.

26 Zum erotischen Potenzial der Formel vgl. ebd., S. 297.

27 Hartmann: Einheit, S. 42.

28 Ebd., S. 29, Anm. 11.

29 Die Strophe II ist als beschreibender Bericht des Sprechers über den Tagesanbruch gestaltet. Das Ich tritt in ihr nicht explizit hervor. Stattdessen erscheint ein Wir (die uns gepar / ein frucht keuschlich zu freuden; II,9f.).

30 plasiniren ist ein Fachwort aus der Wappensprache und heißt „ein Wappen kunstgerecht malen oder erklären“ (Wachinger: Anhang, S. 391). 
Verständnis der Passage nicht zwingend erscheint. ${ }^{31}$ Das wäre aber nötig, um dem Ich Kontur als Dichter-Ich zu verleihen und es eindeutig und ausschließlich an die Autor-Persona zurückzubinden. Auch die Schlussbitte um Erlösung und Beistand in der Todesstunde überrascht lediglich durch die Art ihrer Versprachlichung, nämlich den angedeuteten Kuss zwischen Maria und Ich (III,10 -12), nicht aber in ihrer Zielrichtung. ${ }^{32}$

Grundsätzlich scheint die Ausgestaltung der Sprecher-Instanz als Ich stark funktional bedingt zu sein. Schon die Intimität der konzipierten Sprechsituation macht die Wahl der ersten Person Singular unumgänglich, denn wie sollte sich hier ein kollektives Wir artikulieren? Dass in dem Lied ein Ich spricht, ist demnach zunächst einmal die Konsequenz daraus, dass Oswald Es leucht durch grau die fein lasur als Gattungskontrafaktur zu bestimmten monologischen Sehnsuchtsklagen mit Tageliedmotiven angelegt hat und das Ich als Minnenden inszeniert. ${ }^{33}$ Angesichts der Kühnheit dieses Experiments scheint mir der Verzicht auf eine weitere Konkretisierung des Ich darüber hinaus der eigenen Absicherung gegen den potenziellen Vorwurf der Häresie zu dienen: Indem Oswald das Ich zumindest gegenüber allen männlichen Rezipienten öffnet, macht er sie - gleichsam mit einem Augenzwinkern - zu seinen Komplizen, und es ist insofern womöglich kein Zufall, dass das Lied Erwach an schrick, vil schönes weib (Kl. 40), in dem Oswald dieses Gattungsexperiment wiederholt, ein genauso vage konturiertes Ich aufweist wie das hier interpretierte Kl. 34/Wa. 39. ${ }^{34}$

\subsection{Das Sangspruch-Ich}

Zum dritten Typ zählen solche Lieder, in denen das Ich eine oftmals höchst konventionelle Sprecherrolle einnimmt, die deutlich als Gegenposition zu den Rezipientinnen und Rezipienten konzipiert ist und insofern verhindert, dass der einzelne Rezipient das Ich für sich reklamieren kann. ${ }^{35}$ Eine Abgrenzung der Ich-

31 Bei Oswald selbst taucht die Idee auch in solchen Zusammenhängen auf, in denen keine Einzelstimme, sonder ein Wir spricht. Die Aussage ist dann sicher allgemein zu beziehen; vgl. etwa: „Wer kan volzieren / so genaue / des degens vas, / Das er im selb erwelt?“(Kl. 38/Wa. 38,II,47). Hartmann: Einheit, S. 34f. betont, dass das Marienlob im Spätmittelalter als höchste Aufgabe der Dichter angesehen wurde. Sie würde demzufolge das poetologische Moment eher unterstreichen.

32 Hartmann: Einheit, S. 33, Anm. 31; Wachinger: Blick, S. 303.

33 Wachinger: Blick, S. 307. Dieser Typus des Sehnsuchtslieds ist aber nicht weit verbreitet (ebd.).

$34 \mathrm{Zu}$ dem Lied vgl. Schiendorfer; Spicker, S. 87-89; Wachinger: Blick, S. 300-302.

35 In diese Gruppe gehören die Lieder Kl. 8, Kl. 10, Kl. 11, Kl. 22, Kl. 27, Kl. 32, Kl. 35, Kl. 95, Kl.111, Kl. 112, Kl. 114, Kl. 115 und Kl. 118. 
Position erreicht Oswald einerseits durch die Art und Weise, wie er die Sprechsituation entwickelt. Anders als in den bisher behandelten Liedern kann es bei den Liedern des dritten Typs eine textinterne, in der Regel in der zweiten Person Plural angesprochene Zuhörerschaft (,ihr', ,euch') geben. ${ }^{36}$ Dieses fiktive Publikum fungiert als Identifikationsoption für die realen textexternen Rezipientinnen und Rezipienten. ${ }^{37}$ Zur Abgrenzung des Ich trägt andererseits die spezifische Anlage der Sprecher-Position bei. Das Ich wird nämlich im Anschluss an entsprechende Rollenentwürfe des Sangspruchs als Mahner, Prediger, Ratgeber oder Wissender gezeichnet; ${ }^{38}$ es besitzt demnach stets eine besondere Autorität und einen Wissensvorsprung vor dem Publikum oder den im Lied explizit Angeredeten. Vorherrschend ist ein belehrender Duktus, der die Rezipientinnen und Rezipienten auch dann zu Zuhörern erklärt, wenn das jeweilige Lied keine ,ihr'-Anreden besitzt.

Beispielhaft möchte ich diesen Typ anhand des Weihnachtslieds In Suria ain braiten hall (Kl. 35/Wa. 37) vorführen. ${ }^{39}$ Das Ich meldet sich in diesem Lied an zwei Stellen, nämlich in den Strophen I und III, explizit zu Wort und schlüpft dabei in für den Sangspruch typische Ich-Rollen. Als Augenzeuge verifiziert es in Strophe I die Existenz eines Spalts in der über der Geburtsgrotte errichteten Geburtskirche in Bethlehem. Diese habe sich geöffnet, weil der Teufel über die Geburt des Erlösers in Wut geraten sei:

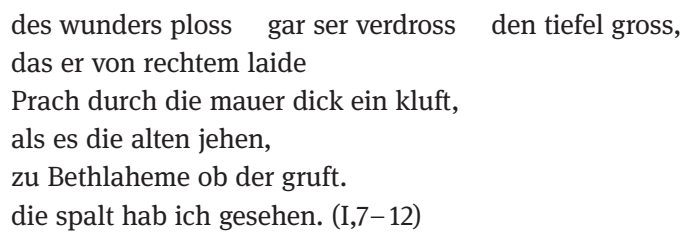

In Strophe III bekennt das hier explizit als ,Wolkenstein' benannte Ich seine Unfähigkeit, die Liebe zwischen Maria und Christus angemessen beschreiben zu

36 In Kl. 10 wird ein einzelner Hörer in der zweiten Person Singular angeredet (Freund, wiltu; Kl. 10,VII,1).

37 Die ,ich-Form muss dann nicht exklusiv sein: Weil mit den angeredeten fiktiven Zuhörern immer schon ein Kollektiv im Text präsent ist, kann das Ich sich partienweise mit dieser Gruppe vereinigen, um sich dann wieder von ihr zu lösen. Dies geschieht etwa in Kl. 8.

38 Der Sangspruch-Bezug wird in der Forschung meistens mit Blick auf Kl. 1-11 angemerkt (vgl. etwa Spicker, S. 103), also für Lieder, die größtenteils nicht zum dritten Typ zählen. Für Kl. 111 verhandelt Schnyder die Frage nach einem meisterlichen Selbstverständnis, bleibt aber skeptisch (Schnyder, S. 47).

39 Vgl. zum Lied Spicker, S. 93 f.; Löser: Geistliche Dichtung, S. 12 f. 
können: das ich Wolkhenstain / die lieb nicht kan beklaiden (III,9f.). Die Formel verbleibt ähnlich stereotyp wie in Kl. 34/Wa. 39, ist aber durch die Namenssignatur eindeutig als poetologische, ausschließlich auf die Autor-Persona beziehbare Aussage gekennzeichnet, die freilich aufgrund ihrer Konventionalität keine besonders große interpretatorische Reichweite hat. Die Signatur ist hier wohl auch inseriert, um einen Rückhalt für die Augenzeugen-Rolle der Strophe I $\mathrm{zu}$ bieten, für die ebenfalls ein biographischer Hintergrund dingfest gemacht werden kann: Normalerweise wird hier eine Reminiszenz an Oswalds Pilgerfahrt ins Heilige Land vermutet. ${ }^{40}$ Die an Christus gerichtete Schlussbitte götleich gepurt, durch magt mentsch rain, / hilf an dem letzten schaiden (III,11f.) ist dann allerdings wieder so offen formuliert, dass jeder als Empfänger der göttlichen Gnade in Frage kommt - das Oswald-Ich bezieht sie eben nicht nur auf sich.

Dass Oswald in diesem und in anderen Liedern des dritten Typs die Abschließung des Ich durch die Namensnennung auf die Spitze treibt, fügt sich zu der für die Gruppe typischen allgemeinen Orientierung am Sangspruch. Um 1400 etabliert sich in dieser Gattung nämlich die Namenssignatur als Möglichkeit, die eigene Autorschaft zu sichern. ${ }^{41}$ Dass Oswald das Verfahren aufgreift, ist gleichwohl in gewisser Weise als Zutat einzustufen und trifft auch nicht auf alle Lieder des dritten Typs zu: ${ }^{42}$ Schon die eingenommene Sprecherrolle an sich impliziert die Abschließung gegenüber den Rezipientinnen und Rezipienten. Weil das Ich als Wissender konzipiert ist und sich dadurch vom Publikum unterscheidet, kann anders als in den zuvor besprochenen Liedern - nicht mehr jeder ,ich“ sagen und dabei eine stimmige Aussage erzeugen. Das Ich ist vielmehr stets mit der Autorkonkretisation gleichzusetzen.

Trotz der Namensnennung wird in Kl. 35/Wa. 37 zudem nur bedingt eine persönliche religiöse Erfahrung rekapituliert. Im Unterschied zu Freimut Löser, der angesichts der beiden ,ich'-Nennungen postuliert, Oswald beziehe „das Heilsgeschehen [...] gleich zweifach auf sich und seine Person“, ${ }^{43}$ würde ich hier anders gewichten und präzisieren. Oswald bezieht ja nicht das direkte Heilsgeschehen auf sich; er thematisiert seine Vermittlerrolle. Das Ich als Autorkonkretisation ist Kundiger und Dichter und kann deswegen Heilsgeschichte nacherzählen, wobei die oben bereits zitierte Bescheidenheitsformel (III,9f.) zusätzlich dazu dient, in der demutsvollen Negation der eigenen Kunstfertigkeit zugleich implizit den Blick auf die literarische Qualität des Lieds zu lenken.

40 Vgl. Spicker, S. 93; Löser: Geistliche Dichtung, S. 13.

41 Vgl. Brunner, S. 402-404.

42 Namensnennungen finden sich in den Liedern Kl. 27, Kl. 35, Kl. 111, Kl. 112, Kl. 115 (Name in der dritten Person) und Kl. 118.

43 Löser: Geistliche Dichtung, S. 13. 


\subsection{Das biographisch konkretisierte Ich}

Zum vierten Typ gehören schließlich all jene Lieder, in denen Oswald das Ich so nachdrücklich mit einer offenbar in großen Teilen an der eigenen Lebensgeschichte orientierten Biographie ausstattet (oder zumindest derart konkrete Details einsetzt), dass das Ich zu einer eigenständigen, gerundeten Sprecher-Figur wird. ${ }^{44}$ Oftmals bilden dabei wiederum aus dem Sangspruch übernommene Sprecherrollen das Fundament für die Ausgestaltung des Ich. ${ }^{45}$ Diese vorgefundenen Rollen werden jedoch mithilfe der biographischen Elemente konkretisiert und damit einzigartig. Obgleich das Ich daher von vornherein in seinen Verweismöglichkeiten so weit eingeschränkt ist, dass es sich nurmehr auf die Oswald-Persona beziehen kann, arbeiten bei Weitem nicht alle Lieder des vierten Typs mit Namensnennungen: Etwa die Hälfte, nämlich sieben von dreizehn Liedern, verzichten auf diese Spezifizierung. ${ }^{46}$

Die alle Lieder dieser Gruppe prägende Verbindung zwischen Konventionalität des der Ich-Gestaltung zugrundeliegenden Rollenentwurfs und biographischer Konkretisierung betrifft auch das Lied Ain anefangk (Kl.1/Wa 22), welches in den drei Oswald-Handschriften A (Wien, ÖNB, cod. 2777, fol. $1^{\mathrm{rv}}$ ), B (Innsbruck, UB, ohne Signatur, fol. $^{\text {rv }}$ ) und c (Innsbruck, Tiroler Landesmuseum Ferdinandeum, F.B. 1950, fol. $1^{\mathrm{r}}-2^{v}$ ) die Sammlung eröffnet. ${ }^{47}$ Das in der letzten Strophe als Wol-

44 In diese Gruppe gehören die Lieder Kl. 1, Kl. 2, Kl. 4-7, Kl. 9, Kl. 12, Kl. 23, Kl. 24, Kl. 36 und Kl. 39. Außerdem zähle ich Kl. 3/Wa. 24 zu dieser Gruppe, freilich als Variation des vierten Typs. Zwar nennt Oswald seinen Namen (der Wolkenstain; III,12), verarbeitet offenbar persönlich Erlebtes (III,10 - 12 mit Hinweis auf die Gefangenschaft und Folter) und macht diese Erlebnisse zum Gegenstand einer Warnung an das Publikum, doch wird das Sprecher-Ich nicht mit dem im Lied erscheinenden ,Wolkensteiner' gleichgesetzt - dieser ist vielmehr eine der vom Sprecher aufgebotenen Exempelfiguren und erscheint so in der dritten Person (vgl. Toepfer, S. 226-230). Orientiert man sich allein an der Gestaltung der Sprecher-Instanz, könnte man Kl. 3/Wa. 24 daher auch dem dritten Typ zuweisen. Sangspruchartige Züge besitzt das Ich der Lieder des vierten Typs allerdings ebenfalls. Ausschlaggebend für meine Zuordnung ist daher der Umstand, dass ich die Einbringung der ,Wolkensteiner'-Figur ähnlich wie Toepfer (vgl. S. 229f.) als Auseinandersetzung mit den autobiographischen Elementen in Oswalds Liedern lese. Basis für die literarische Abweichung, die sich in Kl. 3/Wa. 24 beobachten lässt, sind demnach die Lieder des vierten Typs, in denen das Ich autobiographische Züge trägt.

45 Spicker, S. 103. Der Sangspruch-Bezug zeigt sich auch musikalisch: Ein Teil der Lieder (Kl. 1-7, Kl. 11, Kl. 12) variiert Regenbogens Grauen Ton. Vgl. Hirschberg, Ragotzky, S. 84. Siehe überdies Brunner, S. 404.

46 Namensnennungen finden sich in den Liedern Kl. 1, Kl. 3 (Name in der dritten Person), Kl. 4, Kl. 6, Kl. 7 und Kl. 39.

47 Zum Lied vgl. Hartmann: Altersdichtung, S. 45-85; Hirschberg, Ragotzky, S. 80 - 95; WandWittkowski; Spicker, S. 111-114; Braun, S. 144-146. 
kenstainer (VII,8) benannte Ich spricht hier in der stereotypen Pose des im Alter reuevoll zurückschauenden Mannes, der die weltliche Liebe als sündhaft beklagt. ${ }^{48}$ Ursache für die Desillusionierung und Weltabkehr ist das Verhalten der im Lied namenlos bleibenden Geliebten. Diese hat dem Ich trotz beständiger Dienstbereitschaft (wol dreuzehen jar und dennoch mer / in treuen stät beliben; II,3f.) ihre einstige Zuneigung entzogen und fungiert in der Textgegenwart als seine Kerkermeisterin: Statt von den weißen Armen der Geliebten wird das Ich von eisernen Fesseln umfangen (VI,6-16). Diesen Zustand deutet das Ich als göttliche Strafe für seine sündhafte irdische Liebe (V,7-18). ${ }^{49}$

Kennzeichnend für das Lied ist ein zweifacher Prozess der Konkretisierung. Einerseits überführt Oswald die dem Minnesang entnommene Metapher der Liebesbande in die sinnlich erfahrbare Realität des Ich, da dieses nicht nur im übertragenen Sinn ein Minnegefangener ist, sondern tatsächlich inhaftiert ist und körperlich leidet: ${ }^{50}$

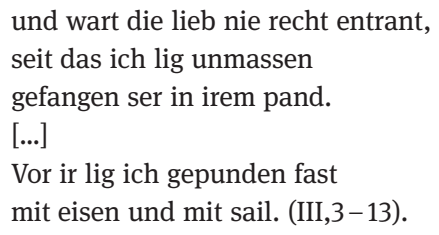

Andererseits formuliert Oswald das Motiv der Gefangenschaft offenbar durchaus unter Rückgriff auf eigene Erlebnisse. Er bezieht sich dabei wahrscheinlich auf die Zeit seiner Inhaftierung während des Besitzstreits um Burg Hauenstein 1421/ $1422 .{ }^{51}$ Vor allem die Plastizität, mit der Oswald die Fesseln des Ich beschreibt, wird in der Forschung als unmittelbarer Reflex dieser Erfahrung gewertet: ${ }^{52}$

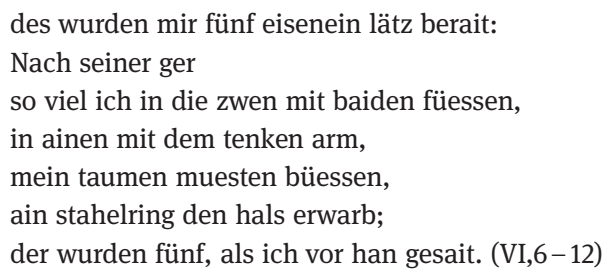

48 Vgl. Hirschberg, Ragotzky, S. 85; Wand-Wittkowski, S. 182f. Zum Typus vgl. Kerth, S. 62.

49 Vgl. Hartmann: Altersdichtung, S. 71-73.

50 Vgl. ebd., S. 49f., Anm. 91 sowie S. 72-75; Hirschberg, Ragotzki, S. 86f.; Wand-Wittkowski, S. 186.

51 Vgl. zum historischen Hintergrund Wand-Wittkowski, S. 181.

52 Vgl. ebd., S. $186 f$. 
Es gibt demnach, wie Braun festhält, „einen biographischen Kern“, der aber nur mehr als „Produktionsanlass für einen Text [erscheint], der ihn ins AllgemeinLiterarische transzendiert und so die biographischen Referenzen schwächt“. ${ }^{53}$ Lieder wie Kl. 1/Wa. 22 gehen mithin nicht im Autobiographischen auf. Vielmehr formt Oswald in ihnen sein eigenes Leben „zum didaktischen Exempel“ um, ${ }^{54}$ wie es das Ich von Kl.1/Wa. 22 direkt als Ziel benennt: da bei ain jeder sol versten, / das lieb an laid die leng nicht mag ergen (I,17 f.). ${ }^{55}$

Neben der figürlichen Ausrundung ist die persönliche Betroffenheit des Ich das zentrale Charakteristikum der Lieder, die sich dem vierten Typ zuordnen lassen. Im Unterschied zu den Liedern des dritten Typs zieht das Sprecher-Ich der Lieder dieser Kategorie seine Lebensgeschichte als Beispiel heran, um eine allgemeine Aussage - etwa wie in Ain anefangk die Notwendigkeit der Abkehr von der Frauenliebe und der Hinwendung zur Gottesliebe - in ihrer Richtigkeit zu belegen. ${ }^{56}$ Dieser Gestus der persönlichen Betroffenheit wird von Oswald biographisch unterfüttert, doch könnte der Effekt ebenso gut durch eine vollständig fiktive Biographie erreicht werden, solange sie detailliert genug dargeboten wird.

\section{Schlussfolgerungen und offene Fragen}

Welchen Nutzen kann man aus der Einteilung der geistlichen Lieder anhand der Ausformung ihrer Sprecher-Instanzen ziehen? Sicherlich ist zunächst einmal die Einsicht ein Gewinn, dass die - die Forschungsdiskussion oftmals dominierenden - geistlichen Lieder mit starker (pseudo)-autobiographischer Prägung von der Art von Ain anefangk (Kl. 1/Wa. 22) nicht stellvertretend für alle geistlichen Lieder Oswalds stehen können. Die enge Konturiertheit der Ich-Position dieser Lieder, die als einzige Deutungsmöglichkeit die Gleichsetzung von Ich und Oswald-Persona gestattet, lässt sich nicht ohne Weiteres auf andere Lieder übertragen, selbst wenn diese ebenfalls in der ersten Person Singular abgefasst sind. Stattdessen gibt es im Oswald-Korpus eine Reihe von Liedern, bei denen das Ich sehr offen gestaltet ist und deswegen zwar potenziell auf die Oswald-Persona

53 Braun, S. 146.

54 Löser: Geistliche Dichtung, S. 22.

55 Vgl. Spicker, S. 112.

56 Dieses Schema der Liedanlage ist bei den Liedern des vierten Typs so verbreitet, dass Oswald es in Kl. 3/Wa. 24 und in Kl. 39/Wa. 35 ironisch-spielerisch variieren kann. Diese Variationen lassen sich jeweils als poetologischer Kommentar lesen, weil Oswald sie nutzt, um auf die Distanz zwischen Sprecher-Ich und realem Autor hinzuweisen (vgl. Toepfer, insbes. S. 236f.). 
beziehbar bleibt, ebenso gut aber als eine Art ,kollektives‘ Ich die Rezipientinnen und Rezipienten meinen könnte.

Ein Unsicherheitsfaktor in der Debatte bleibt jedoch die in der Oswald-Forschung immer noch umstrittene Frage nach der Rezeption der Lieder. ${ }^{57}$ Ich habe sie bislang weitestgehend ausgeblendet und mich zunächst mit Bedacht allein auf die Beschreibung der textuell vorgenommenen Ausgestaltung der Sprecher-Instanz konzentriert, weil zu einer abschließenden Beurteilung der Aufführungssituation und ihrer Einwirkung auf das Verständnis des Ich zuverlässige Daten fehlen. Für die Lieder des ersten und zweiten Typs, deren offen angelegte Ichs durch jedermann gefüllt werden können, ist die Rezeptionsform indes womöglich der ausschlaggebende Aspekt, um auf Basis der konkreten Rezeptionserfahrung eine bestimmte Auslegung des Ich zu favorisieren. Denn dass bei den genannten Liedtypen unterschiedliche Referentialisierungen des Ich (Bezug auf die UrheberInstanz oder auf die Rezipienten) denkbar wären, beschreibt lediglich das Potenzial, das im Text angelegt ist. In der konkreten Rezeptionssituation wird sich der einzelne Rezipient, die einzelne Rezipientin für ein bestimmtes Verständnis entscheiden. So läge es in einer Vortragssituation wahrscheinlich eher nahe, das Ich der Texte mit dem Vortragenden zu identifizieren, während in der privaten Lektüre der jeweilige Rezipient das Ich vielleicht primär auf sich selbst bezieht. Je nach Anlass und zugänglicher Rezeptionsform sind die betreffenden Lieder daher vielleicht verschieden verstanden worden.

Jenseits dieser Unsicherheiten kann man zwei wichtige Ergebnisse festhalten. Einerseits bestätigt der Versuch, die verschiedenen Sprecher-Instanzen zu kategorisieren, wie funktional ihre Ausgestaltung auf die einzelnen Lieder abgestimmt ist. Das sieht man bereits daran, dass die an die Liturgie oder den Ritus angenäherten geistlichen Lieder auf ein Ich verzichten und stattdessen ein Wir sprechen lassen, denn schon diese grundsätzliche Entscheidung impliziert, dass Oswald der Benutzung der ersten Person Singular oder Plural eine bestimmte Bedeutung beimisst. Auch in der relativ kleinen Gruppe der erotischen Marienlieder (zweiter Typ) ist deren recht konturlose Ich-Gestaltung virtuos darauf abgestimmt, die Minnendenrolle aufzunehmen, die Intimität der Sprechsituation zu wahren und gleichzeitig eindeutige biographische Zuspitzungen zu vermeiden, die angesichts der Brisanz des Formexperiments vielleicht unakzeptabel wären.

57 Umstritten ist vor allem, ob die Lieder ausschließlich als Leselieder rezipiert worden sind oder ob es auch Aufführungen gegeben hat, bei denen Oswald die Lieder vorgetragen hat. Vgl. zur Diskussion Mertens, S. 342f. Mertens selbst geht von einer vorhandenen, aber nicht sehr vitalen Aufführungstradition aus; die Lieder seien wohl eher als Buchlyrik zu verstehen und die durch die Melodiebeigaben evozierte Deutung als Aufführungslieder sei eine Inszenierung (ebd.). 
Andererseits relativiert die vorgestellte Typologie die Bedeutung des Biographischen für Oswalds geistliche Lieder, und dies nicht nur, weil so jene Lieder hervortreten, die ohne biographische Elemente auskommen. Vielmehr wird auf diese Weise vor allem deutlich, dass die autobiographische Ausschmückung des Ich gleichsam sekundär ist. Oswald kreiert Sprechsituationen, in denen das Ich nurmehr mit der Autor-Persona gleichgesetzt werden kann, indem er bei den Liedern des dritten Typs dem Ich bestimmte Rollen zuweist, die den Zuhörern unzugänglich sind, und ihnen dafür mit dem im Text angesprochenen fiktiven Publikum ein alternatives Identifikationsangebot macht. In den Liedern des vierten Typs versieht er das Ich mit einer Biographie, die es figürlich ausrundet. Beides sind literarische Strategien der Konkretisierung und Abschließung, die auch dann aufgehen, wenn die hierzu benutzten Informationen (Name, biographische Elemente etc.) nichts mit dem historischen Autor zu tun haben. Dass Oswald seine eigene Biographie literarisch verarbeitet, mag dem Zeitgeist oder den veränderten Rezeptionsbedingungen geschuldet sein, ${ }^{58}$ ist aber zunächst einmal seine spezifische Art, sich bestimmte, im späteren Mittelalter auch sonst virulente literarische Darstellungsmuster anzueignen.

\section{Literaturverzeichnis}

Braun, Manuel: Lebenskunst oder: Namen als biographische Referenzen bei Oswald von Wolkenstein. In: JOWG 19 (2012/2013), S. 137-162.

Brunner, Horst: Tradition und Innovation im Bereich der Liedtypen um 1400. Beschreibung und Versuch der Erklärung. In: Textsorten und literarische Gattungen. Dokumentation des Germanistentages in Hamburg vom 1. bis 4. April 1979. Berlin 1983, S. 392-413.

Die Lieder Oswalds von Wolkenstein. Hg. von Karl Kurt Klein. 4., grundlegend neu bearbeitete Aufl. von Burghart Wachinger. Berlin, Boston 2015 (ATB 55).

Glier, Ingeborg: Artes amandi. Untersuchungen zur Geschichte, Überlieferung und Typologie der deutschen Minnereden. München 1971 (MTU 34).

Haferland, Harald: Frühe Anzeichen eines lyrischen Ichs. Zu einem Liedtyp der gedruckten Liedersammlungen aus der ersten Hälfte des 16. Jahrhunderts (Erhart Öglin, Peter Schöffer, Arnt von Aich, Christian Egenolff, Heinrich Finck, Georg Forster, Johann Ott). In: Deutsche Liebeslyrik im 15. und 16. Jahrhundert. 18. Mediävistisches Kolloquium des Zentrums für Mittelalterstudien der Otto-Friedrich-Universität Bamberg am 28. und 29. November 2003. Hg. von Gert Hübner. Amsterdam, New York 2005 (Chloe 37), S. $169-200$.

58 Meistens wird in diesem Kontext angenommen, dass der Übergang zum Leselied eine Konkretisation der Sprecher-Instanz durch Biographisierungstechniken notwendig gemacht habe, weil das Ich nicht mehr auf einen Sänger projiziert werden konnte und insofern seinen Ankerpunkt verlor. Vgl. Mertens, S. 343. 
Hartmann, Sieglinde: Altersdichtung und Selbstdarstellung bei Oswald von Wolkenstein. Die Lieder Kl 1 bis 7 im spätmittelalterlichen Kontext. Göppingen 1980 (GAG 288).

Hartmann, Sieglinde: Zur Einheit des Marienliedes Kl. 34. Eine Stilstudie mit Übersetzung und Kommentar. In: JOWG 3 (1984/1985), S. 25- 43.

Hartmann, Sieglinde: Gotische Madonnenbilder und die Marienlyrik Oswalds von Wolkenstein. In: wort unde wîse, singen unde sagen. Fs. für Ulrich Müller zum 65. Geburtstag. Hg. von Ingrid Bennewitz. Göppingen 2007 (GAG 741), S. 71-92.

Hausmann, Albrecht: Reinmar der Alte als Autor. Untersuchungen zur Überlieferung und zur programmatischen Identität. Tübingen, Basel 1999 (Bibliotheca Germanica 40).

Hirschberg, Dagmar, Hedda Ragotzky: Zum Verhältnis von Minnethematik und biographischer Realität bei Oswald von Wolkenstein: Ain anefangk (Kl. 1) und Es fügt sich (Kl. 18). In: JOWG 3 (1984/1985), S. 79-114.

Kerth, Sonja: myn heubt daz ist mir worden gra / myn ruck hat sich gebogen. Alter(n) in der Sangspruchdichtung von Reinmar von Zweter bis Michel Beheim. In: JOWG 21 (2016/2017), S. 59-72.

Kraß, Andreas: Sünder, Prediger, Dichter: Rollenspiele im Beichtlied Oswalds von Wolkenstein. In: Punishment and Penitential Practices in Medieval German Writing. Hg. von Sarah Bowden, Annette Volfing. London 2018, S. 65-78.

Löser, Freimut: Oswald von Wolkenstein: Geistliche Lieder. In: Oswald von Wolkenstein. Leben - Werk - Rezeption. Hg. von Ulrich Müller, Margarete Springeth. Berlin, New York 2011, S. 251-261.

Löser, Freimut: Oswalds von Wolkenstein geistliche Dichtung. In: JOWG 19 (2012/2013), S. 5-30.

Marold, Werner: Kommentar zu den Liedern Oswalds von Wolkenstein. Im Anschluss an die Ausgabe von Jos. Schatz (1902/1904). Teil 1, 2. http://www-gewi.uni-graz.at/marold/ index.html. Diss. ms. Göttingen 1926 (28. August 2019).

Mertens, Volker: ,Biographisierung in der spätmittelalterlichen Lyrik. Dante - Hadloub Oswald von Wolkenstein. In: Kultureller Austausch und Literaturgeschichte im Mittelalter. Kolloquium im Deutschen Historischen Institut Paris 16.-18.3.1995. Hg. von Ingrid Kasten, Werner Paravicini, René Pérennec. Sigmaringen 1998 (Beihefte der Francia 43), S. $331-344$.

Oswald von Wolkenstein: Lieder. Frühneuhochdeutsch/Neuhochdeutsch. Ausgewählte Texte hg., übersetzt und kommentiert von Burghart Wachinger. Melodien und Tonsätze hg. und kommentiert von Horst Brunner. Stuttgart 2007 (RUB 18490).

Oswald von Wolkenstein: Sämtliche Lieder und Gedichte. Ins Neuhochdeutsche übersetzt von Wernfried Hofmeister. Göppingen 1989 (GAG 511).

Philipowski, Katharina: Autodiegetisches Erzählen in der mittelhochdeutschen Literatur oder: Warum mittelalterliche Erzähler singen müssen, um von sich erzählen zu können. In: ZfdPh 132 (2013), S. 321-351.

Schiendorfer, Max: Ain schidlichs streuen. Heilsgeschichte und Jenseitsspekulation in Oswalds verkanntem Tagelied KI 40. In: JOWG 9 (1996/1997), S. 179-196.

Schnyder, André: Das erste Passionslied Oswalds von Wolkenstein - Eine Interpretation von KI 111 (mit einem Seitenblick auf G 23 des Mönchs von Salzburg). In: Speculum medii aevi 2 (1996), S. 43-58.

Spechtler, Franz Viktor: Beiträge zum deutschen geistlichen Lied des Mittelalters II: Oswald von Wolkenstein. In: Oswald von Wolkenstein. Beiträge der philologisch- 
musikwissenschaftlichen Tagung in Neustift bei Brixen 1973. Hg. von Egon Kühebacher. Innsbruck 1974 (Innsbrucker Beiträge zur Kulturwissenschaft. Germanistische Reihe 1), S. $272-284$.

Spechtler, Franz Viktor: Beiträge zum deutschen geistlichen Lied des Mittelalters III: Liedtraditionen in den Marienliedern Oswalds von Wolkenstein. In: Gesammelte Vorträge der 600-Jahrfeier Oswalds von Wolkenstein Seis am Schlern 1977. Hg. von Hans-Dieter Mück, Ulrich Müller. Göppingen 1978 (GAG 206), S. 179-203.

Spicker, Johannes: Oswald von Wolkenstein. Die Lieder. Berlin 2007 (Klassiker-Lektüren 10). Toepfer, Regina: Oswald von Wolkenstein und sein Sprecher-Ich. Poetisches Spiel mit autobiographischen Elementen in den Liedern KI 3, 33 und 39. In: JOWG 19 (2012/2013), S. 225-240.

Wachinger, Burghart: Art. ,Oswald von Wolkenstein‘. In: VL² 7 (1989), Sp. 134-169.

Wachinger, Burghart: Anhang. In: Oswald von Wolkenstein. Lieder. Frühneuhochdeutsch/ Neuhochdeutsch. Ausgewählte Texte hg., übersetzt und kommentiert von Burghart Wachinger. Melodien und Tonsätze hg. und kommentiert von Horst Brunner. Stuttgart 2007 (RUB 18490), S. 313-423.

Wachinger, Burghart: Blick durch die braw. Maria als Geliebte bei Oswald von Wolkenstein [2001]. In: Ders.: Lieder und Liederbücher. Gesammelte Aufsätze zur mittelhochdeutschen Lyrik. Berlin, New York 2011, S. 297-310.

Wand-Wittkowski, Christine: Topisches oder biographisches Ich? Das Lied Ain anefangk Oswalds von Wolkenstein. In: Wirkendes Wort 52 (2002), S. 178-191. 
